\title{
Design of Magazine Reading System Based on Android
}

\author{
（WeiguoZou，Yancheng Institute of Industry Technology, Yancheng 224001, China )
}

\begin{abstract}
By mobile internet's development, the technology of mobile and mobile terminal, digitized function change with each passing day, environmental protection of paperless needs to be break. The digital magazine reading system based on Android OS developed in this study has independent intellectual property, and is leading Chinese digital news changing in the past years..
\end{abstract}

KEYWORDS: Magazine Reading System; Electronization; Android

\section{Introduction}

With the popularization of intelligent mobile terminal platform, and further implementation of green environmental protection concept advocated by country, for timeliness, economy, communication, environmental protection and other aspects, traditional print media has reflected growing gap with current social development. Cultural content distribution based on the Internet, mobile phones and other new forms of media has become mainstream. Changes of the way people read, access to information and knowledge, and even their lifestylehave taken place in subversive.

The technology to show print media content through mobile and computer terminals, has changed the inherent business model of paper media "one-way communication", so readers can be more timely, accurate and easy access to the information they want. The era of mass communication is about to shift to era of personalized communication. The realization of electronic transmission of paper mediafundamentally solves the problem of tree felling, papermaking, printing, issuance, pollutionand other environmental issues.

The ultimate goal of the topic is to develop magazine reading software based on Android OS. This system mainly realizes paper magazine "authentic" browsing on the WEB, digital magazine retrieval, digital magazine static page file generation and release, etc. By entering the PDF files, PS files and image files and other formats files, the operations of inverse solution, indexing, recognition, custom editing, etc. are performed, and then the digital magazine is generated, and will be published to the terminal equipment of Android platform so that anyone can browse and view it.

\section{Overall Design}

\subsection{System Overall Framework}

This system is composed of three parts of media management terminal program, server program and mobile terminal. After electronization of the paper magazine content, the media management terminal will transmit and store it in the server. According to the demand, mobile and computer terminals request the server to transmit the electronic magazine content to the terminals. Then, the terminal programs resolve the data and echo it. The overall framework of the magazine reading system is shown in Fig. 1: 


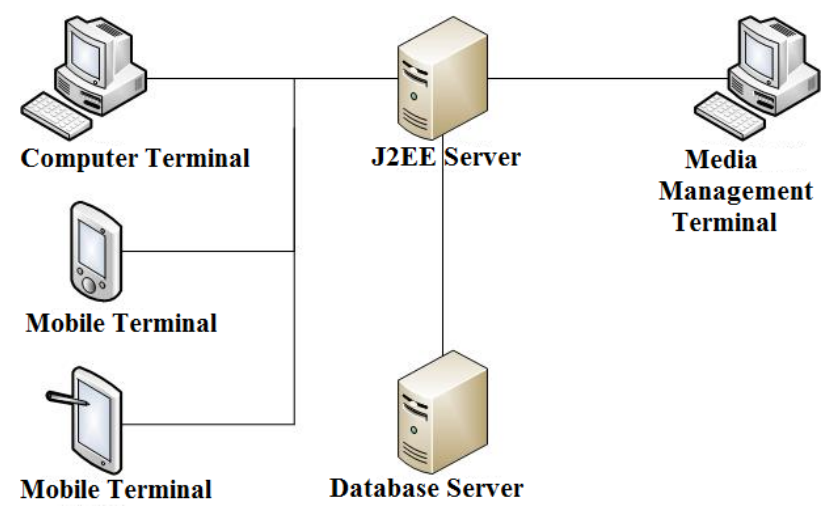

Fig.1. Overall Framework of the System

\subsection{System Architecture}

According to hierarchy, the system can be divided into three layers of application layer, business logic layer and database layer. The specific description of the architecture is shown in Fig. 2:

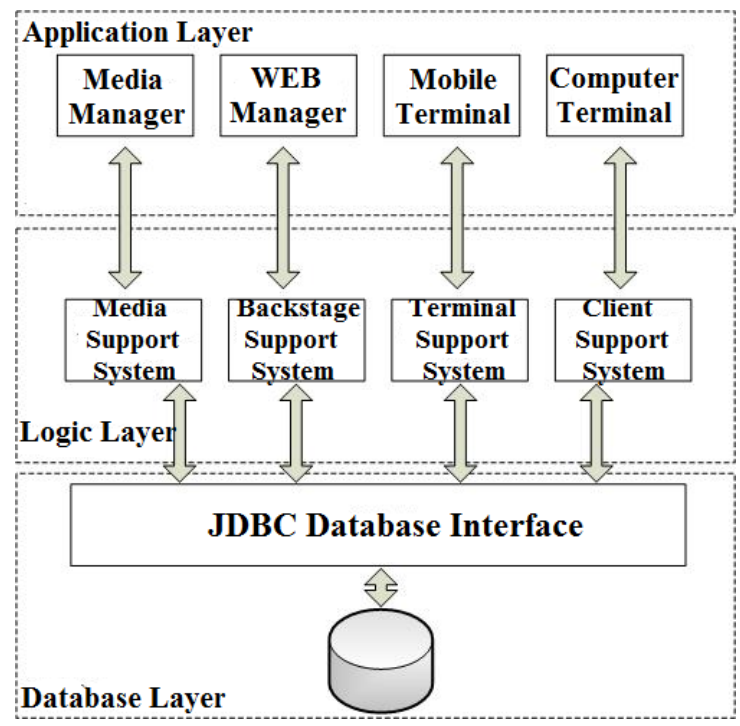

Fig.2. Architecture of the System

(1) Application Layer

a) Media management terminal: with client computer as the main realization way, it is responsible for electronization and normalization of paper magazine data, and then submit to the corresponding business logic module to store. b) Back-stage management terminal: based on browser, its main function is to manage the media data and the terminal data in the browser.

c) Mobile terminal: based on different mobile terminal platform to develop the clients, mainly realize magazine content echo in the mobile terminals. 
d) Computer terminal: based on browser, its main function is to display the electronic magazine contents in the browser.

(2) Business Logic Layer

Corresponding to the modules in application layer, business logic layer organizes and transmits the data requested by each module.

(3) Database Layer

Database layer mainly is mainly responsible for basic data storage system and read function

\section{Detailed Design}

\subsection{Functional Design}

This system mainly realizes the functions of PDF, FS and image files interpretation, articles secondary editing, articles management, HTML digital magazine generation and digital magazine cross-platform release, etc. The functional modules design is shown in Fig. 3:

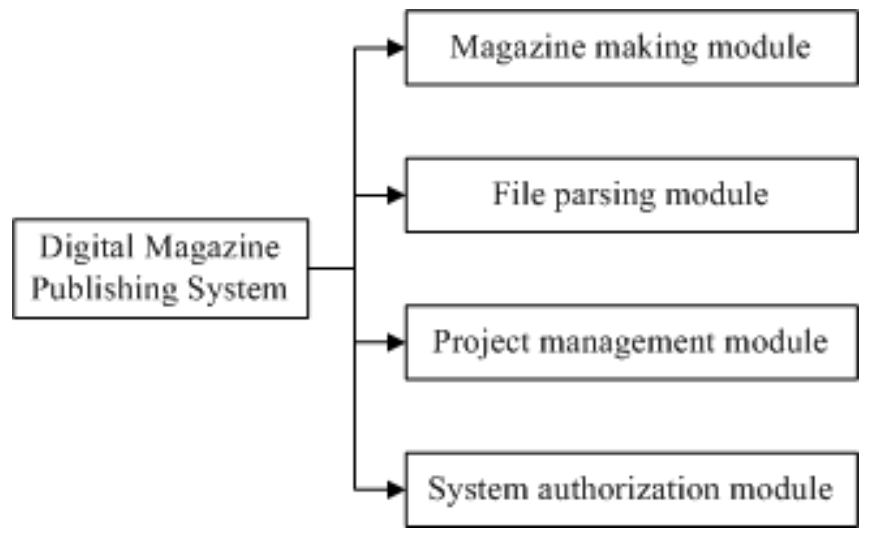

Fig.3. Functional Modules of the System

\subsection{Magazine Making Module}

Magazine making module is the main module of digital magazine reading system, including three sub-modules of intelligent text blocking, digital magazine editing and digital magazine generation. After importing and parsing of the file, the pictures in every layout and the layout corresponding text message are showed in the form of text blocks. The functions of magazine making module are as follow:

(1) Layout indexing: mark the pictures and text of the file in different colors, including article headlines, leading title, subtitle, pictures, text, author and other information, for the convenience of the user to edit the articles.

(2) OCR character recognition and correction: in order to import pictures to the project, we need to make OCR character recognition. After that, we still need to do manual correction and correct the recognition errors in the text, so that ensure the correct identification rate.

(3) Text analysis and intelligent blocking: merge the single text blocks into text block, title block, text block, author block, etc.

(4) Article management: synthesize the various properties indexed in the layout, so each layout is corresponding to one or more articles. This function can achieve various management operations of the articles, such as adding, modification, deleting articles.

(5) Image conversion: it includes conversion and storage of the pictures in each layout. The pictures need to be converted into different sizes so that they can be used under different circumstances, such as OCR pictures, mobile 
terminal pictures, static pages pictures and project display pictures, etc.

(6) Templates importing: read the template files into the program, and then according to the templates produce similar static HTML files.

\subsection{File Parsing Module}

PS/PDF file parsing system is essentially a pre-processing module of essentially a pre-processing module PS/PDFfile parsing indexing publishing system, which paves the way for the indexing and publishing after parsing. PS/PDF file parsing system saves the parsed page information in the form of an electronic page, and transfers it to publishing system for further processing, and finally forming electronic publications. The detailed workflow of PS/PDF file parsing system is shown in Fig. 4:

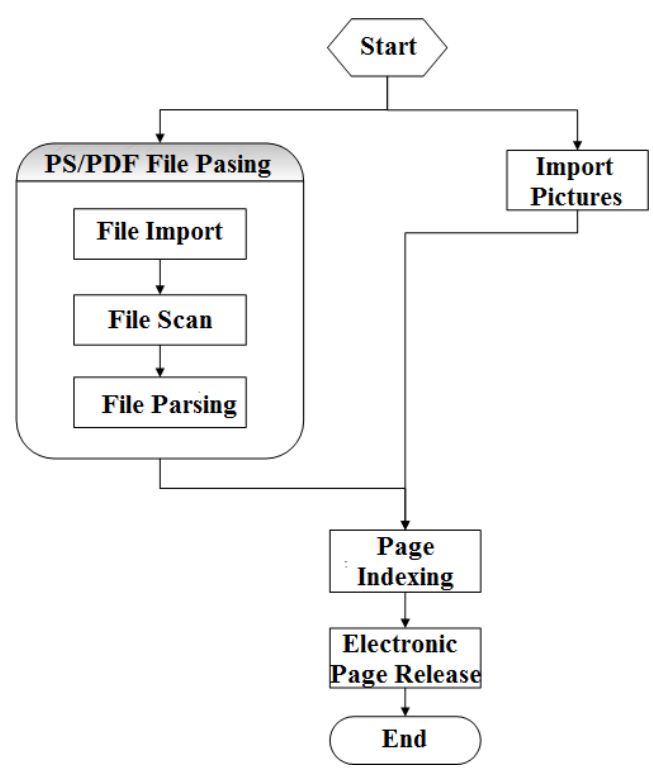

Fig.4. Workflow of File Parsing System

\subsection{Project Management Module}

In the system, it manages the relevant data of digital magazine by Project DM. So system should provide user with rich project management functions, including project newly-built, project configuration, project opening, project elimination, and project close.

(1) Project newly-built: to build a new project, we need to provide project name, project catalogue and other basic information, which is stored by way of project properties.

(2) Project configuration: configure the relevant information of the project, such as OCR setting and so on.
(3) Project opening: open the project by selecting an existing project file, by which can avoid some configuration operation and reuse the existing project.

(4) Project elimination: in the process of project execution, we can eliminate the data files, programs and some project configuration information in the testing process, and restore the testing process to the initial state.

(5) Project close: suspend the currently executing process and close the project. 


\subsection{System Authorization Module}

The system provides two ways for users to register: user name registration and license registration.

(1) User name registration: users actively enter the phone number and password, and send SMS to Register.

(2) License registration: users login in the digital magazine reading client, and establish registrationrelationship with background.

User access control mechanism is introduced into the system.In order to overcome the defects of mandatory access control and Discretionary access control, we introduce the role based access control model- RBAC model. The model connects the users and permissions, to control user access to system resources based on role authorization.

\section{Conclusion}

In view of the current global magazine paperless issuance trends, we have developed a digital magazine distribution platform, saving a lot of printing cost and manpower cost. The system can help publish the cooperative magazines in mobile and computer terminal within a few minutes, which is fast, convenient, aging and environmental. The digital magazine reading system is leading the Chinese media in the digital transition.

\section{REFERENCES:}

[1] Rosenfeld A, Kak A C. Digital picture processing[M]. Elsevier, 2014.

[2] Gonzalez R. Multimedia publishing system for wireless devices: U.S. Patent Application 10/498,558[P]. 2002-12-13.

[3] Nagasaka A, Tanaka Y. Automatic video indexing and full-video search for object appearances[J]. 1992.

[4] Ferrel P J, Meyer R F, Millet S J, et al. Method for delivering separate design and content in a multimedia publishing system: U.S. Patent 6,199,082[P]. 2001-3-6.

[5] Santos Silva D. The future of digital magazine publishing[J]. Information Services and Use, 2011, 31(3): 301-310.

[6] Schwartz D, Kenny P. Method of producing and delivering an electronic magazine in full-screen format: U.S. Patent Application 10/828,252[P]. 2004-4-21. 